\title{
Perceived ability to perform daily hand activities after stroke and associated factors: a cross-sectional study
}

\author{
Elisabeth Ekstrand ${ }^{1,2^{*}}$ (D), Lars Rylander ${ }^{3}$, Jan Lexell ${ }^{1,4,5}$ and Christina Brogårdh ${ }^{1,4}$
}

\begin{abstract}
Background: Despite that disability of the upper extremity is common after stroke, there is limited knowledge how it influences self-perceived ability to perform daily hand activities. The aim of this study was to describe which daily hand activities that persons with mild to moderate impairments of the upper extremity after stroke perceive difficult to perform and to evaluate how several potential factors are associated with the self-perceived performance.
\end{abstract}

Methods: Seventy-five persons (72\% male) with mild to moderate impairments of the upper extremity after stroke (4 to 116 months) participated. Self-perceived ability to perform daily hand activities was rated with the ABILHAND Questionnaire. The perceived ability to perform daily hand activities and the potentially associated factors (age, gender, social and vocational situation, affected hand, upper extremity pain, spasticity, grip strength, somatosensation of the hand, manual dexterity, perceived participation and life satisfaction) were evaluated by linear regression models.

Results: The activities that were perceived difficult or impossible for a majority of the participants were bimanual tasks that required fine manual dexterity of the more affected hand. The factor that had the strongest association with perceived ability to perform daily hand activities was dexterity $(p<0.001)$, which together with perceived participation ( $p=0.002$ ) explained $48 \%$ of the variance in the final multivariate model.

Conclusion: Persons with mild to moderate impairments of the upper extremity after stroke perceive that bimanual activities requiring fine manual dexterity are the most difficult to perform. Dexterity and perceived participation are factors specifically important to consider in the rehabilitation of the upper extremity after stroke in order to improve the ability to use the hands in daily life.

Keywords: Activities of daily living, Association, Cross-sectional study, Rehabilitation, Stroke, Self report, Upper extremity

\section{Background}

Disability of the upper extremity is common after stroke and almost $50 \%$ of those affected have remaining impairments more than three months post-stroke $[1,2]$. The impairments often lead to difficulties in performing daily hand activities [3], especially those that require the use of both hands, i.e., bimanual activities [4]. The ability to perform bimanual activities is therefore an important

\footnotetext{
* Correspondence: elisabeth.ekstrand@med.lu.se

'Department of Health Sciences, Lund University, Lund, Sweden

${ }^{2}$ Department of Hand Surgery, Skåne University Hospital, Malmö, Sweden

Full list of author information is available at the end of the article
}

goal in stroke rehabilitation, regardless of which hand that is affected [5].

The ability to perform daily activities can be objectively assessed by observations of different tasks in a standardized environment or by patient-reported questionnaires. The advantage of using questionnaires is that they often provide a better understanding of an individual's self-reported everyday difficulties and thereby enable clinicians to design more individually targeted rehabilitation interventions [6]. One questionnaire that is recommended for persons with disability of the upper extremity after stroke is the ABILHAND Questionnaire 
$[4,7,8]$. It assesses self-perceived ability to perform daily bimanual activities. Previous studies have focused on evaluating the psychometric properties of the ABILHAND $[4,8]$, but no study has thoroughly described which activities persons in a stable phase post stroke perceive difficult to perform.

In order to improve functioning of the upper extremity after stroke, it is important to understand which factors affect self-perceived ability to perform daily hand activities. Previous studies have shown that single factors, such as motor function, muscle strength, spasticity, somatosensation, dexterity, perceived participation and life satisfaction are moderately to strongly associated with the perceived ability [4, 9-17]. However, as several factors simultaneously may influence the ability to perform daily hand activities there is a need to understand how these factors are associated with the performance. To the best of our knowledge, only one study [14] has evaluated this association in persons in a stable phase after stroke. In that study by Harris and Eng [14], muscle strength, spasticity, somatosensation and pain were included in multivariate analyses and the authors found that muscle strength in the upper extremity and spasticity were the strongest contributing factors to the perceived ability to use the hands in daily activities. However, dexterity was omitted as a potentially associated factor in the analysis, which was addressed as a limitation of the study. In other studies, gender, dominance of the affected upper extremity, and social and vocational situations have been shown to be important factors for overall functioning after stroke [18-21]. However, it is unclear how these factors are associated with the self-perceived ability.

Taken together, despite that disability of the upper extremity is common after stroke there is limited knowledge of which daily activities that are perceived difficult to perform and which factors that affect the self-perceived performance. The majority of previous studies have evaluated how single or few factors are associated with perceived daily hand activities. Thus, there is a need for more studies that take several factors into account simultaneously.

The aim of this study was to evaluate a) which daily activities persons with mild to moderate impairments of the upper extremity after stroke perceive difficult to perform and b) how several factors (age, gender, social and vocational situation, affected hand, upper extremity pain, spasticity, grip strength, somatosensation, manual dexterity, perceived participation and life satisfaction) are associated with the self-perceived performance.

\section{Methods}

\section{Participants}

Persons diagnosed with stroke (ischemic or hemorrhagic) and admitted to the stroke unit at the Department of
Neurology and Rehabilitation Medicine at Skåne University Hospital in the southern part of Sweden, were recruited from April 2012 to August 2015. They were identified through cooperation with physiotherapists and occupational therapists working in the stroke rehabilitation centres serving the acute neurology wards at Skåne University Hospital. The inclusion criteria were: (i) $>18$ years of age; ii) at least 4 months after stroke onset; (iii) mild to moderate impairments of the more affected upper extremity with preserved ability to take the palm to the forehead, and to grasp and release a small object. Persons were excluded if: (i) they were unable to follow instructions or (ii) had any other disorder or disease that affected the more affected upper extremity.

\section{Outcome measures}

Perceived ability to perform daily hand activities was rated with the ABILHAND Questionnaire (stroke version) [4]. The ABILHAND consists of 23 common bimanual activities (see Table 2 ) that are rated as impossible (0 point), difficult (1 point) or easy ( 2 points). Items not attempted within the last three months are set as not applicable. The items are ordered hierarchically, from the most difficult items to the easiest, and they are also rated according to the level of bimanual involvement: $\mathrm{A}=$ breakable into unimanual sequences; $\mathrm{B}=$ requires stabilization with the more affected upper extremity; and $\mathrm{C}=$ requires fine bimanual dexterity [4]. The ABILHAND is Rasch analyzed [4], which means that ordinal data can be converted into an unidimensional interval scale, and presented in logits (i.e., log odds units) that ranges from plus to minus around zero as the center of the scale [22]. The higher the logit value, the better the self-perceived ability to use the upper extremities in daily hand activities. In this study, the Swedish version of the ABILHAND was used [23], which has been shown to have acceptable test-retest reliability for persons with mild to moderate impairments of the upper extremities after stroke [8]. Due to cultural differences the item 'Peeling potatoes with a knife' was changed to 'Peeling potatoes' (as a potato-peeler is commonly used in Sweden) and in the item 'Tearing open a pack of chips' the following words were added 'or a candy-bag' (because older persons in Sweden more rarely eat chips) [8]. After the participants had responded to the ABILHAND the logits were obtained by entering the raw scores into an online data analysis module (http://www.rehab-scales.org/) established for chronic stroke patients [4].

Pain (present or not) was recorded by asking the participants if they perceived daily or almost daily pain in their more affected upper extremity.

Spasticity was assessed by the response to resistance of passive movement according to the Modified Ashworth Scale (MAS) [24]. The participants' spasticity was assessed in an upright sitting position and was classified as present 
if the elbow, wrist or fingers had a score on the MAS larger or equal to 1 point. The MAS has been shown to have high intra-rater reliability of the upper extremity for persons with stroke [25].

Grip strength was measured with the digital dynamometer Grippit (Catell AB, Hägersten, Sweden, http://www. catell.se/). The Grippit is a portable device that is wirelessly connected to a computer. Grip strength was measured three times with the participants seated with the forearm supported on a table on a foam cushion (the shoulder in $30^{\circ}$ flexion, the elbow in $90^{\circ}$ flexion and the wrist in $0^{\circ}$ to $15^{\circ}$ dorsiflexion) according to a standardized test protocol [26]. Each contraction lasted $3 \mathrm{~s}$ with a $60 \mathrm{~s}$ rest interval between each repetition. The highest value in Newton $(\mathrm{N})$ of the three contractions was recorded as the maximal grip strength. Measurements of grip strength with the Grippit dynamometer have been shown to have an acceptable test-retest reliability for persons with mild to moderate impairments of the upper extremity after stroke [26], and grip strength has also been found to be a representative measure of the entire upper extremity muscle strength after stroke [27].

Active touch (somatosensation) of the hand was assessed with the The Shape/Texture Identification test (STI-test) [28] (Össur Nordic AB, Uppsala, Sweden, http://www. ossur.se/). Active touch means that identification of different shapes and textures is done by active hand movements. Compared to passive touch, active touch has the advantage of reflecting how somatosensation is integrated during hand movements. The STI-test includes three shapes (cube, cylinder or hexagon) and three textures (one, two or three raised metal dots placed in a row) in three difficulty levels (decreasing sizes). According to the standardized test instructions [28], the participants were seated behind a screen and identified the shapes (presented randomly size for size) by the index finger. Thereafter the textures were presented and identified in the same way. The score of the STI-test ranges from 0 to 6 points per hand and a higher score indicates better somatosensation $[28,29]$. The STI-test has been shown to have high test-retest reliability for persons with mild to moderate impairments of the upper extremity after stroke [29].

Dexterity was assessed by the modified Sollerman Hand Function test (mSHFT) [30] (Catell AB, Hägersten, Sweden, http://www.catell.se/). The mSHFT assesses manual dexterity by common pinch and volar grips. It is a short version of the 20-item original Sollerman Hand Function Test [31, 32] and consists of the three items most strongly correlated with the total score [30]. The items in the mSHFT are: number 4) picking up 4 coins of different sizes from a purse; number 8 ) putting 4 nuts in decreasing size on bolts; and number 10) buttoning 4 buttons in decreasing sizes. These items are performed as unimanual tasks and the ability to grasp the object correctly, the time to complete the item and the quality of the movement are both assessed on a 5-point scale ( 0 to 4 points). The total sum score ranges between 0 and 12 points for each hand (where 12 approximates normal dexterity) [30]. The mSHFT has been found to be valid and reliable for persons with mild to moderate impairments of the upper extremity after stroke [33].

Perceived participation, i.e., a person's engagement in meaningful life situations, was rated by the participation domain of the Stroke Impact Scale 3.0 (SIS; Swedish version), that can be used as a separate scale $[34,35]$. SIS Participation is interview-based and includes eight items: impact of stroke on work, social activities, quiet recreations, active recreations, role as a family member, religious activities, life control and ability to help others. The items are scored on a 5-point scale from 1 (limited all of the time) to 5 (limited none of the time). The mean for the items is calculated as a composite score and converted into a percentage value (from 0 to 100) [35], and a higher percentage value indicates higher perceived participation. SIS has been shown to be reliable and valid in persons with stroke [34,36].

Life Satisfaction was rated by the Life Satisfaction Questionnaire (LiSat-11) [37]. LiSat-11 is interview-based and includes one item that assesses the level of global satisfaction with life as a whole and 10 items that assess the level of domain-specific satisfaction. In the present study, only the item of global satisfaction with life as a whole was used. The responses were rated on a six-graded scale: $6=$ very satisfied; $5=$ satisfied; $4=$ rather satisfied; $3=$ rather dissatisfied; $2=$ dissatisfied; and $1=$ very dissatisfied. In this study, the score was dichotomized into two categories; dissatisfied (score 1 to 4 ) and satisfied (5 and 6) according to Fugl-Meyer et al. [37].

\section{Procedures}

Prior to the assessments the participants were asked about their age, handedness, social situation (if they lived alone or together with another person) and their vocational situation (not working or in work at least $20 \mathrm{~h}$ per week).

The assessments were performed in the following order: 1) perceived pain 2) ability to perform daily hand activities (ABILHAND) [4]; 3) spasticity (MAS) [24]; 4) perceived participation (SIS-Participation) [34]; 5) dexterity (mSHFT) [30]; 6) active touch (STI-test) [28]; 7) life satisfaction (LiSat-global satisfaction) [37]; and 8) grip strength (Grippit) [26]. Each test took about $10 \mathrm{~min}$ to complete and a short rest (approximately $5 \mathrm{~min}$ ) was allowed between the tests. All assessments were performed on one occasion according to the standardized test procedures of each test in a quiet and separate room of the hospital by an experienced physiotherapist (first author). Data on time of stroke onset, type of stroke 
(ischemic or hemorrhagic) and side of paresis were verified from the medical records.

\section{Statistics}

Descriptive statistics, such as frequencies, means and standard deviations (SD) and medians and minimum and maximum (min-max) were calculated for demographic and clinical characteristics of the participants, perceived ability to perform daily hand activities (logits) and potentially associated factors. The distribution of the participants' ratings (easy, difficult, impossible or not applicable) of the 23 items in the ABILHAND was presented in percent.

Before the linear regression analyses were conducted correlations between the ordinal and continuous potentially associated factors were calculated using the Spearman correlation (rho) to investigate the strength of their associations.

Perceived ability to perform daily hand activities (continuous: logits) and the potentially associated factors were analyzed by linear regression models. The potentially associated factors were: age (continuous); gender (categorical: female $v s$ male); social situation (categorical: living together with another $v s$ living alone); vocational situation (categorical: working $v s$ not working); affected hand (categorical; dominant $v s$ non-dominant); perceived pain in the more affected upper extremity (categorical: present vs not present); spasticity in the more affected upper extremity (categorical: present $v s$ not present); grip strength in the more affected hand (continuous: newton); active touch in the more affected hand (ordinal scale; 0 to 6 points); dexterity in the more affected hand (ordinal scale; 0 to 12 points), perceived participation (ordinal scale; 0 to $100 \%$ ) and life satisfaction (categorical: satisfied $v s$ dissatisfied).

The multivariate regression building was made with a generous inclusion criterion $(p \leq 0.20)$ so that no potential variable was excluded in the early stages. First, the associations with perceived ability to perform daily hand activities were evaluated for one variable at the time. Secondly, the variable with the lowest $p$-value (if $\leq 0.20$ ) was kept and thereafter the other variables were tentatively added, one at a time. Thirdly, the two variables with the lowest $p$-values (if both $\leq 0.20$ ) were included and the remaining factors were tentatively added, one at a time. This procedure was continued as long as the $p$-values for all included factors were $\leq 0.20$. This selection strategy, to evaluate the model at each step, was chosen as it increases the understanding of the different variables significance.

The explained variances (adjusted $R^{2}$ ) after successive addition of the factors are given in the final multivariate model. To ensure the linearity, scatterplots were visually inspected for the bivariate associations. In addition, model assumptions were checked by means of residual analysis.
Data were analyzed with the IBM SPSS Statistics version 22 (IBM Corporation, Armonk, New York, United States).

\section{Results \\ Demographics}

Out of 270 potential participants, 75 persons with ischemic $(n=58)$ or hemorrhagic $(n=17)$ stroke were included in the study (Fig. 1 and Table 1). The mean time from stroke onset was 33 months (SD 26; range 4 to 116). The participants (72\% male) were on average 66 years (SD 8; range 44 to 85 ), one third lived alone and most of them did not work. About half of the participants were affected in their dominant hand.

\section{Description of perceived ability to perform daily hand activities}

Table 2 shows the distribution of the ratings (ordinal data) of the 23 bimanual items in the ABILHAND in a hierarchical order. Between 41 and $61 \%$ of the participants perceived the following eight items difficult or impossible to perform: 'filing one's nails'; 'hammering a nail'; 'cutting meat'; wrapping up gifts'; 'threading a needle'; 'tearing open a pack of chips'; 'buttoning up a shirt'; and 'cutting one's nails'. All but one of these items except for 'buttoning up a shirt' were classified as requiring fine manual dexterity of the more affected hand (level C) [4]. Thirteen items were perceived as easy to perform by most of the participants. Those that only required stabilization with the more affected hand (level B) were perceived as easy by 60 to $84 \%$ of the participants, and those that could be breakable into unimanual sequences (level A) were perceived as easy by 63 to $95 \%$ of the participants. Two of the items shelling hazel nuts'and 'sharpening a pencil' (level C) were not attempted within the last 3 months for a majority of the participants and therefore noted as not applicable.

\section{Perceived ability to perform daily hand activities and associated factors}

Table 3 presents a summary of data regarding perceived ability to perform daily hand activities and the potentially

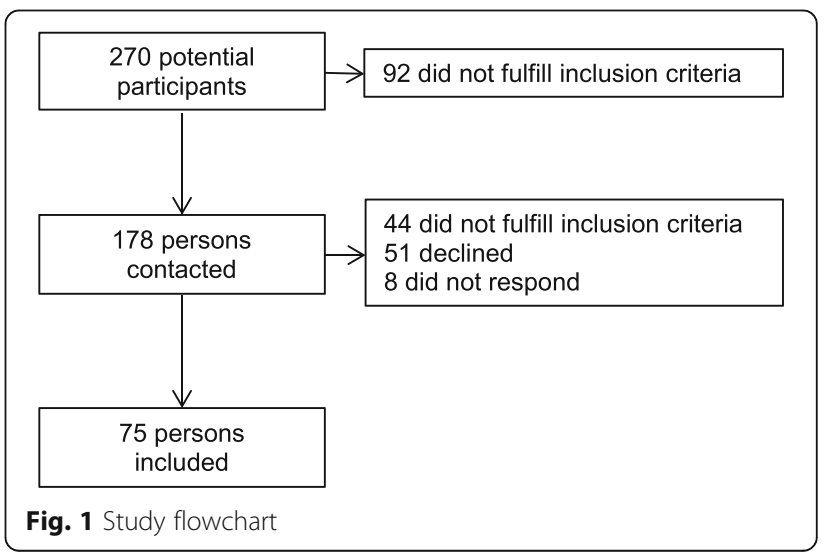


Table 1 Characteristics of the 75 participants with stroke

\begin{tabular}{ll}
\hline Age, mean years (SD; range) & $66(8 ; 44$ to 85) \\
Gender (male), n (\%) & $54(72)$ \\
Time since stroke, mean months (SD; range) & $33(26 ; 4$ to 116) \\
Stroke type, n (\%) & $58(77)$ \\
$\quad$ Ischemic & $17(23)$ \\
$\quad$ Hemorrhagic & $37(49)$ \\
Side of paresis (right), n (\%) & $39(52)$ \\
Affected hand (dominant), n (\%) & $21(28)$ \\
Social situation (living alone), n (\%) & $62(83)$ \\
Vocational situation (not working), n (\%)
\end{tabular}

associated factors (i.e., pain and spasticity in the more affected upper extremity; grip strength, active touch and dexterity in the more affected hand; perceived participation and life satisfaction).

\section{Correlation analyses}

Table 4 shows the bivariate correlations among the potentially associated factors treated as continuous or ordinal variables. The correlations were generally low (rho $<0.5$ ) except for the correlation $(\mathrm{rho}=0.68)$ between dexterity and active touch (somatosensation).

\section{Univariate regression analysis}

Table 5 presents the univariate associations between perceived ability to perform daily hand activity (logits) and potentially associated factors obtained from the univariate linear regression models. The factor that showed the strongest association with perceived ability to perform daily hand activities was dexterity $\left(R^{2}=0.39, p<0.001\right)$. A one unit increase in dexterity corresponded to 0.32 increased logits of the ABILHAND score (i.e., a $\beta$-coefficient of 0.32 ). The associations between age, social situation, vocational situation, spasticity, grip strength, active touch, perceived participation and life satisfaction, respectively, and perceived ability to perform daily hand activities fulfilled the criteria for being included in the multivariate analyses (i.e., $p$-values $\leq 0.20$ ).

\section{Multivariate regression analyses}

Table 6 presents the three factors that were associated with the perceived ability to perform daily hand activity

Table 2 Distribution (\%) of ratings in the ABILHAND Questionnaire $(n=75)$

\begin{tabular}{|c|c|c|c|c|c|c|}
\hline & Items & Impossible & Difficult & Easy & NA & $\overline{\text { Level }}$ \\
\hline$\overline{1}$ & Hammering a nail & 19 & 28 & 31 & 23 & $C$ \\
\hline 2 & Threading a needle & 23 & 31 & 15 & 32 & C \\
\hline 3 & Peeling potatoes & 13 & 35 & 51 & 1 & C \\
\hline 4 & Cutting one's nails & 19 & 42 & 39 & 0 & C \\
\hline 5 & Wrapping up gifts & 16 & 35 & 23 & 27 & C \\
\hline 6 & Cutting meat & 9 & 41 & 48 & 1 & C \\
\hline 7 & Filing one's nails & 8 & 33 & 31 & 28 & C \\
\hline 8 & Peeling onions & 12 & 29 & 51 & 8 & C \\
\hline 9 & Shelling hazel nuts & 0 & 9 & 20 & 71 & C \\
\hline 10 & Opening a screw-topped jar & 5 & 23 & 71 & 1 & C \\
\hline 11 & Fastening the zipper of a jacket & 3 & 37 & 60 & 0 & B \\
\hline 12 & Tearing open a pack of chips & 15 & 41 & 40 & 4 & C \\
\hline 13 & Buttoning up a shirt & 12 & 45 & 41 & 1 & A \\
\hline 14 & Sharpening a pencil & 0 & 4 & 41 & 55 & C \\
\hline 15 & Taking the cap off a bottle & 1 & 21 & 76 & 1 & B \\
\hline 16 & Spreading butter on a slice of bread & 1 & 27 & 72 & 0 & A \\
\hline 17 & Fastening a snap (jacket, bag) & 3 & 12 & 84 & 1 & $\mathrm{~B}$ \\
\hline 18 & Buttoning up trousers & 4 & 29 & 65 & 1 & $\mathrm{~B}$ \\
\hline 19 & Opening mail & 0 & 6 & 84 & 0 & B \\
\hline 20 & Pulling up the zipper of trousers & 2 & 16 & 80 & 1 & A \\
\hline 21 & Squeezing tooth-paste on a toothbrush & 0 & 8 & 92 & 0 & A \\
\hline 22 & Unwrapping a chocolate bar & 1 & 32 & 63 & 4 & A \\
\hline 23 & Washing one's hands & 0 & 5 & 95 & 0 & A \\
\hline
\end{tabular}

Items ranked from 1 to 23: from more difficult to less difficult. NA not applicable. Level A: the item is breakable into unimanual sequences; level B: the item requires stabilization with the affected limb; level $C$ : the item requires fine bimanual dexterity. Due to truncation the percentage values do not add up to $100 \%$ for all items 
Table 3 Summary of the measurements $(n=75)$

\begin{tabular}{|c|c|}
\hline $\begin{array}{l}\text { Daily hand activities (ABILHAND logits), } \\
\text { mean (SD) }\end{array}$ & $2.0(S D 1.7)$ \\
\hline Upper extremity pain (present), n (\%) & $32(43)$ \\
\hline Spasticity (present), n (\%) ${ }^{a}$ & $23(31)$ \\
\hline Grip strength (newton), mean (SD) ${ }^{b}$ & $198(110)$ \\
\hline $\begin{array}{l}\text { Active touch ( } 0 \text { to } 6 \text { points), mean } \\
(\mathrm{SD}) / \text { median }(\text { min-max) }\end{array}$ & $3.8(2.2) / 5$ (0 to 6$)$ \\
\hline $\begin{array}{l}\text { Dexterity (0 to } 12 \text { points), mean (SD)/median } \\
(\text { min-max) })^{d}\end{array}$ & $5.4(3.3) / 5$ (0 to 12$)$ \\
\hline $\begin{array}{l}\text { Participation (0 to } 100 \% \text { ), mean (SD)/median } \\
\left(_{(\text {min-max })^{e}}\right.\end{array}$ & $69(19) / 67$ (12 to 100$)$ \\
\hline Life satisfaction (dissatisfied), n (\%) ${ }^{f}$ & $35(47)$ \\
\hline
\end{tabular}

(logits) in the final multivariate linear regression model: dexterity $(p<0.001)$, perceived participation $(p=0.002)$ and grip strength $(p=0.180)$. The explained variance was $39 \%$ for dexterity, which increased to $48 \%$ when perceived participation was added to the model. Grip strength only increased the explained variance with $1 \%$, but was added due to the generous inclusion criterion $(p \leq 0.20)$. The $\beta$-coefficient for dexterity changed from 0.32 in the univariate model to 0.26 in the multivariate model and the corresponding change for perceived participation (per 10 units increase) was 0.39 to 0.26 .

\section{Discussion}

The main findings of this study were that bimanual tasks requiring a high level of fine bimanual dexterity were perceived most difficult to perform. Dexterity was the factor that had the strongest association with perceived ability to perform daily hand activities and explained together with perceived participation $48 \%$ of the variance in the final multivariate model.

\section{Description of perceived ability to perform daily hand activities}

The ratings of the items in the ABILHAND revealed that eight of the 23 items were perceived difficult or impossible to perform. All of those items were bimanual

Table 4 Bivariate correlations (rho) between ordinal and continuous factors $(n=75)$

\begin{tabular}{lllll}
\hline & Age & Grip strength & Active touch & Dexterity \\
\hline Grip strength & $-0.21^{\mathrm{a}}$ & & & \\
Active touch & -0.03 & $0.24^{\mathrm{a}}$ & & \\
Dexterity & -0.19 & $0.46^{\mathrm{b}}$ & $0.68^{\mathrm{b}}$ & \\
Participation & -0.18 & $0.24^{\mathrm{a}}$ & 0.21 & 0.21 \\
\hline
\end{tabular}



${ }^{\mathrm{b}}$ Correlation is significant at the 0.01 level tasks classified as requiring a high level of fine manual dexterity (level C) [4] except for the item 'buttoning up a shirt'. Many of the participants expressed that they could manage the buttons on the chest, but the ones on the sleeves were difficult or impossible to perform with the more affected hand. Thus, even if this item is classified as breakable into unimanual sequences (level A) this suggests that it actually requires fine manual dexterity of the more affected hand (level C). Moreover, the item 'peeling potatoes' is classified as a task requiring fine bimanual dexterity (level C) [4] but was considered as easy for a majority of the participants in our study. Peeling potatoes in Sweden is normally done with a special potato-peeling tool instead of a knife that is suggested in the original version of the questionnaire [4], and this could possibly explain why this item was rated as easy. The other items that were rated as easy were primarily breakable into unimanual sequences (level A) or only required stabilization with the affected upper extremity (level B). As ability to perform daily hand activities is an important goal in the rehabilitation after stroke [5] the ratings of the items in the ABILHAND could be helpful for the patients in their goal setting. Moreover, the different levels (A, B and C) may be useful for the clinicians in the analysis of daily hand activities regarding the bimanual involvement and dexterity demands. The underlying sensorimotor functions required for the tasks can then be specifically practiced in order to achieve the patient's goals.

\section{Perceived ability to perform daily hand activities and associated factors}

In the present study, the association between perceived ability to perform daily hand activities and several factors were analysed. All factors, except for upper extremity pain, gender and affected hand, were included in the multivariate analyses. Dexterity of the more affected hand was strongest associated with perceived ability to perform daily hand activities. Manual dexterity includes the ability to execute coordinated hand and finger movements when grasping, manipulating and releasing objects. Reduced dexterity can result in impaired grip formation and independent finger movements, and in reduced timing and force regulation of the hand [38, 39]. As manual dexterity is important for upper extremity functioning it should be thoroughly assessed and intensively practiced during stroke rehabilitation to enhance the use of the hands in daily activities.

In the final multivariate model, perceived participation was included as the second strongest factor and added another $9 \%$ of the explained variance. This underscores the importance of considering a person's engagement in meaningful life situations in stroke rehabilitation. Traditionally, most of the rehabilitation is carried out 
Table 5 Results from the univariate linear regression models $(n=75)$

\begin{tabular}{|c|c|c|c|}
\hline Determinants & $R^{2}$ & $p$-value & $\beta$-value $(95 \% \mathrm{Cl}$ ) \\
\hline Age (per year increase) & 0.04 & 0.11 & $-0.04(-0.09$ to 0.01$)$ \\
\hline Gender (female vs male [ref]) & 0.00 & $0.60^{\#}$ & $-0.23(-1.10$ to 0.63$)$ \\
\hline Social situation (living together vs living alone [ref]) & 0.04 & 0.07 & $0.78(-0.10$ to 1.62$)$ \\
\hline Vocational situation (working vs not working [ref]) & 0.04 & 0.11 & $0.82(-0.19$ to 1.82$)$ \\
\hline Affected hand (dominant vs non-dominant [ref]) & 0.01 & $0.50^{\#}$ & $0.26(-1.04$ to 0.51$)$ \\
\hline Pain (present vs not present [ref]) & 0.01 & $0.51^{\#}$ & $-0.26(-1.04$ to 0.52$)$ \\
\hline Spasticity (present vs not present [ref]) ${ }^{a}$ & 0.08 & 0.01 & $-1.04(-1.84$ to -0.23$)$ \\
\hline Grip strength (newton, per 10 units increase) & 0.17 & $<0.001$ & 0.06 (0.03 to 0.09$)$ \\
\hline Active touch (per unit increase) ${ }^{c}$ & 0.23 & $<0.001$ & 0.37 (0.21 to 0.52$)$ \\
\hline Dexterity (per unit increase) ${ }^{d}$ & 0.39 & $<0.001$ & 0.32 (0.24 to 0.41$)$ \\
\hline Participation (per 10 units increase) ${ }^{e}$ & 0.19 & $<0.001$ & 0.39 (0.20 to 0.57$)$ \\
\hline Life satisfaction (satisfied vs dissatisfied [ref] $)^{f}$ & 0.09 & 0.01 & $0.98(0.24$ to 1.72$)$ \\
\hline
\end{tabular}

"The variable did not fulfil the criteria, $p \leq 0.20$, for being included in the continued multivariate evaluations; ref: reference group (indicate the category to which the other category is compared). Data obtained by ${ }^{a}$ the Modified Ashworth Scale; ${ }^{b}$ the Grippit dynamometer; ${ }^{c}$ the Shape/Texture Identification Test; ${ }^{d}$ the modified Sollerman Hand Function Test; ${ }^{e}$ the Stroke Impact Scale domain Participation; and ${ }^{f}$ the Life Satisfaction Questionnaire (life as a whole)

in the early phase after stroke. However, it has been shown in a recent large European multicenter study that the arm function and activity performance for persons with stroke deteriorate over time [40]. This indicates that longer follow-up periods and interventions in the later phases after stroke are needed, with emphasis on regaining and finding new ways to participate in meaningful activities in order to maintain the ability to perform daily hand activities over time.

Furthermore, in our final multivariate model grip strength was included as the third factor, but added only $1 \%$ to the explained variance. In the study by Harris and Eng [14] both arm and grip strength were associated with the ability to perform daily hand activities. Arm strength was the strongest contributor in their final multivariate model and together with spasticity and grip strength they explained $93 \%$ of the variance in daily hand activities. In the present study, we used grip strength as a proxy for muscle strength of the entire upper extremity, as grip strength has been shown to be highly correlated to shoulder and elbow muscle strength after stroke [27]. Dexterity was not included in the study by Harris and Eng as they included persons with more severe impairments in the upper extremity after stroke. Thus, our results are difficult to fully compare with that study. Altogether though, the findings indicate that manual dexterity is more important for daily hand activities in persons with milder impairments, whereas strength is more important for persons with severe impairments.

Despite that we included several potentially associated factors in our multivariate analyses, dexterity and perceived participation were the only significant contributors in the final model and together they explained $48 \%$ of the variance of perceived ability to perform daily hand activities. Even though this is a high degree of association, it still suggests that other factors may be important to consider in the rehabilitation of persons with mild to moderate impairments of upper extremity after stroke and that daily hand activities need to be measured and trained per se.

Somatosensation (i.e., active touch) was the factor that showed the second highest correlation with perceived ability to perform daily hand activities. However, active touch was not included in the final multivariate model

Table 6 The final multivariate linear regression model $(n=75)$

\begin{tabular}{|c|c|c|c|}
\hline Factors & $\beta$-value $(95 \% \mathrm{Cl}$ ) & $p$-value & Explained variance $^{d}(\%)$ \\
\hline Dexterity (per unit increase) ${ }^{a}$ & $0.26(0.16$ to 0.35$)$ & $<0.001$ & \\
\hline Participation (per 10 units increase) ${ }^{b}$ & $0.26(0.10$ to 0.42$)$ & 0.002 & \\
\hline Grip strength (newton, per 10 units increase) ${ }^{c}$ & $0.02(-0.01$ to 0.05$)$ & 0.180 & \\
\hline Dexterity & & & 39 \\
\hline Dexterity + participation & & & 48 \\
\hline Dexterity + participation + grip strength & & & 49 \\
\hline
\end{tabular}

Data obtained by: ${ }^{a}$ the modified Sollerman Hand Function Test; ${ }^{b}$ the Stroke Impact Scale domain Participation; and ${ }^{\mathrm{c}}$ the Grippit dynamometer. ${ }^{\mathrm{d}}$ Explained variances after successive addition of determinants 
due to the high correlation with dexterity. This suggests that to be able to perform manual dexterity tasks somatosensation is an important underlying factor. Somatosensation, measured as passive touch, has in a previous study [41] been found to correlate significantly with dexterity in persons with mild impairments in the upper extremity after stroke. Future studies should therefore investigate how different modalities of somatosensation are associated with gross and fine manual dexterity and the influence on the performance of daily hand activities after stroke.

\section{Strengths and limitations}

A strength of the present study was that the participants with mild to moderate upper extremity impairments after stroke were recruited from an unselected population living both in urban and non-urban areas in the southern part of Sweden. The participants were all in a stable phase post-stroke, care was taken to standardize the test situation and one examiner performed all assessments. Stroke specific outcome measures were used to assess functioning and disabilities of the upper extremity, valid and reliable for persons with stroke. Moreover, the multivariate model included several factors in order to evaluate their association with perceived ability to use the upper extremities in daily activities after stroke.

The sample size in the present study was based on a reasonable number of persons judged to be sufficiently large to evaluate the associations of interest. However, for weak associations a larger study sample is required. Only persons with mild to moderate impairments of upper extremity were included in the present study, and those with major cognitive impairments or difficulties to communicate were excluded. More men volunteered to participate and the results can therefore not be generalized to the entire stroke population. Furthermore, subgroup analyses to evaluate differences regarding gender, dominant or non-dominant affected hand and time since stroke would have been interesting, but were not conducted as we judged the sample size being too small. Moreover, it cannot be excluded that other factors may be of importance for the ability to perform daily hand activities after stroke, for example vision, cognitive functions, fatigue, self-efficacy, aids, family and health care support. These variables were not included in the present study as too many variables may cause fatigue in the assessments of persons with stroke.

As this was a cross-sectional study we cannot state that the causality directly results from the factors included in the regression models. Prospective studies are therefore needed in order to evaluate how several potentially associated factors influence the perceived ability to perform daily hand activities in persons with mild to moderate impairments of upper extremity after stroke over time.

\section{Conclusion}

This cross-sectional study showed that the daily hand activities that were perceived difficult or impossible to perform were tasks requiring a high level of fine bimanual dexterity. Dexterity was the strongest contributor to perceived ability to perform daily hand activities and together with perceived participation explained $48 \%$ of the variance in the final multivariate model. This suggests that dexterity and participation are particularly important to consider in the rehabilitation of upper extremity after stroke. The explained variance implies that other factors may also be of importance to improve the ability to use the hands in daily life after stroke.

\section{Abbreviations \\ Cl: Confidence interval; LiSat-11: Life Satisfaction Questionnaire; MAS: Modified Ashworth Scale (MAS); mSHFT: modified Sollerman Hand Function Test; SD: Standard deviation; SIS: Stroke Impact Scale; STI-test: Shape/Texture Identification test}

\section{Acknowledgements}

The authors are grateful to the individuals who volunteered to participate.

\section{Funding}

The study was supported by grants from, the Swedish Stroke Association, Vårdakademin at Skåne University Hospital, the Norrbacka Eugenia Foundation and Skåne county council's research and development foundation.

\section{Availability of data and materials}

All relevant data are presented in the manuscript. Our raw data are coded and the key is available only to the responsible researchers. The Research Ethics Committee at Lund University has not given permission to let anyone else deal with the data. If requested, by contacting the corresponding author, Elisabeth Ekstrand, we can give more information concerning the background of our statistical computations.

\section{Authors' contributions}

$E E$, $L R$, JL and CB conceived and designed the study. CB obtained funding and ethical approval. EE collected the data, performed the statistical analysis, with support from $L R$, and wrote the article. $C B, L R$ and $J$ revised the article. All authors read and approved the final manuscript.

\section{Competing interests}

The authors declare that they have no competing interests.

\section{Consent for publication}

Not applicable.

\section{Ethics approval and consent to participate}

All participants were given verbal and written information about the study prior to inclusion and gave their written informed consent to participate. The principles of the Declaration of Helsinki were followed and approval was obtained by the Regional Ethical Review Board, Lund, Sweden

(Dnr 2012/591).

\section{Author details}

${ }^{1}$ Department of Health Sciences, Lund University, Lund, Sweden. ${ }^{2}$ Department of Hand Surgery, Skåne University Hospital, Malmö, Sweden. ${ }^{3}$ Division of Occupational and Environmental Medicine, Lund University, Lund, Sweden. ${ }^{4}$ Department of Neurology and Rehabilitation Medicine, Skåne University Hospital, Lund, Sweden. ${ }^{5}$ Department of Health Science, Luleå University of Technology, Luleå, Sweden.

Received: 11 June 2016 Accepted: 25 October 2016 Published online: 02 November 2016 


\section{References}

1. Persson HC, Parziali M, Danielsson A, Sunnerhagen KS. Outcome and upper extremity function within 72 hours after first occasion of stroke in an unselected population at a stroke unit. A part of the SALGOT study. BMC Neurol. 2012;12:162.

2. Broeks JG, Lankhorst GJ, Rumping K, Prevo AJ. The long-term outcome of arm function after stroke: results of a follow-up study. Disabil Rehabil. 1999;21:357-64.

3. Dobkin BH. Clinical practice. Rehabilitation after stroke. N Engl J Med. 2005;352:1677-84

4. Penta M, Tesio L, Arnould C, Zancan A, Thonnard JL. The ABILHAND questionnaire as a measure of manual ability in chronic stroke patients: Rasch-based validation and relationship to upper limb impairment. Stroke. 2001;32:1627-34.

5. Waddell KJ, Birkenmeier RL, Bland MD, Lang CE. An exploratory analysis of the self-reported goals of individuals with chronic upper-extremity paresis following stroke. Disabil Rehabil. 2016;38:853-7.

6. Stewart JC, Cramer SC. Patient-reported measures provide unique insights into motor function after stroke. Stroke. 2013:44:1111-6.

7. Alt Murphy M, Resteghini C, Feys P, Lamers I. An overview of systematic reviews on upper extremity outcome measures after stroke. BMC Neurol. 2015;15:29.

8. Ekstrand $\mathrm{E}$, Lindgren I, Lexell J, Brogardh C. Test-retest reliability of the ABILHAND questionnaire in persons with chronic stroke. PM R. 2014;6:324-31.

9. Li KY, Lin KC, Wang TN, Wu CY, Huang YH, Ouyang P. Ability of three motor measures to predict functional outcomes reported by stroke patients after rehabilitation. NeuroRehabilitation. 2012;30:267-75

10. Fleming MK, Newham DJ, Roberts-Lewis SF, Sorinola IO. Self-perceived utilization of the paretic arm in chronic stroke requires high upper limb functional ability. Arch Phys Med Rehabil. 2014;95:918-24.

11. Rand D, Eng JJ. Predicting daily use of the affected upper extremity 1 year after stroke. J Stroke Cerebrovasc. 2015;24:274-83.

12. Basilio ML, de Faria-Fortini I, Polese JC, Scianni AA, Faria CD, Teixeira-Salmela LF. Handgrip strength deficits best explain limitations in performing bimanual activities after stroke. J Phys Ther Sci. 2016;28:1161-5.

13. Bae JH, Kang SH, Seo KM, Kim DK, Shin HI, Shin HE. Relationship between grip and pinch strength and activities of daily living in stroke patients. Ann Rehabil Med. 2015:39:752-62.

14. Harris JE, Eng JJ. Paretic upper-limb strength best explains arm activity in people with stroke. Phys Ther. 2007;87:88-97.

15. Bertrand AM, Mercier C, Bourbonnais D, Desrosiers J, Gravel D. Reliability of maximal static strength measurements of the arms in subjects with hemiparesis. Clin Rehabil. 2007;21:248-57.

16. Welmer AK, Holmqvist LW, Sommerfeld DK. Limited fine hand use after stroke and its association with other disabilities. J Rehabil Med. 2008:40:603-8.

17. Bouffioulx E, Arnould C, Thonnard JL. Satisfaction with activity and participation and its relationships with body functions, activities, or environmental factors in stroke patients. Arch Phys Med Rehabil. 2011;92:1404-10.

18. Petrea RE, Beiser AS, Seshadri S, Kelly-Hayes M, Kase CS, Wolf PA. Gender differences in stroke incidence and poststroke disability in the Framingham heart study. Stroke. 2009:40:1032-7.

19. Harris JE, Eng JJ. Individuals with the dominant hand affected following stroke demonstrate less impairment than those with the nondominant hand affected. Neurorehabil Neural Repair. 2006;20:380-9.

20. Jellema S, van der Sande R, van Hees S, Zajec J, Steultjens EM, Nijhuis-van der Sanden MW. Role of environmental factors on resuming valued activities poststroke: a systematic review of qualitative and quantitative findings. Arch Phys Med Rehabil. 2016;97:991-1002.

21. Palmcrantz S, Widen Holmqvist L, Sommerfeld DK. Young individuals with stroke: a cross sectional study of long-term disability associated with self-rated global health. BMC Neurol. 2014;14:20.

22. Rasch G. Probabilistic models for some intelligence and attainment tests. Expanded ed. Chicago: University of Chicago Press; 1980.

23. Gustafsson S, Stibrant Sunnerhagen K, Dahlin-ivanoff S. Occupational therapists' and patients' perceptions of ABILHAND, a new assessment tool for measuring manual ability. Scand J Occup Ther. 2004;11:107-17.

24. Bohannon RW, Smith MB. Interrater reliability of a modified Ashworth scale of muscle spasticity. Phys Ther. 1987:67:206-7.
25. Gregson JM, Leathley M, Moore AP, Sharma AK, Smith TL, Watkins CL. Reliability of the Tone Assessment Scale and the modified Ashworth scale as clinical tools for assessing poststroke spasticity. Arch Phys Med Rehabil. 1999;80:1013-6.

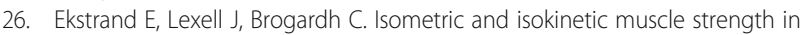
the upper extremity can be reliably measured in persons with chronic stroke. J Rehabil Med. 2015:47:706-13.

27. Ekstrand E, Lexell J, Brogardh C. Grip strength is a representative measure of muscle weakness in the upper extremity after stroke. Top Stroke Rehabil. 2016:4:1-6.

28. Rosén $B$, Lundborg G. A new tactile gnosis instrument in sensibility testing. J Hand Ther. 1998;11:251-7.

29. Ekstrand E, Lexell J, Brogardh C. Test-retest reliability of the Shape/Texture Identification testTM in people with chronic stroke. Clin Rehabil. 2015. [Epub ahead of print]

30. Rosén B. Recovery of sensory and motor function after nerve repair. A rationale for evaluation. J Hand Ther. 1996:9:315-27.

31. Sollerman C, Ejeskar A. Sollerman hand function test. A standardised method and its use in tetraplegic patients. Scand J Plast Reconstr Surg Hand Surg. 1995:29:167-76.

32. Brogårdh $\mathrm{C}$, Persson $\mathrm{AL}$, Sjolund $\mathrm{BH}$. Intra- and inter-rater reliability of the Sollerman hand function test in patients with chronic stroke. Disabil Rehabil. 2007;29:145-54

33. Ekstrand $\mathrm{E}$, Lexell J, Brogardh $\mathrm{C}$. Test-retest reliability and convergent validity of three manual dexterity measures in persons with chronic stroke. PM R. 2016. doi:10.1016/j.pmrj.2016.02.014. [Epub ahead of print].

34. Duncan PW, Bode RK, Min Lai S, Perera S. Rasch analysis of a new stroke-specific outcome scale: the Stroke Impact Scale. Arch Phys Med Rehabil. 2003;84:950-63.

35. Duncan PW, Wallace D, Lai SM, Johnson D, Embretson S, Laster LJ. The stroke impact scale version 2.0. Evaluation of reliability, validity, and sensitivity to change. Stroke. 1999;30:2131-40.

36. Lai SM, Perera S, Duncan PW, Bode R. Physical and social functioning after stroke: comparison of the Stroke Impact Scale and Short Form-36. Stroke. 2003:34:488-93.

37. Fugl-Meyer AR, Melin R, Fugl-Meyer KS. Life satisfaction in 18- to 64-year-old Swedes: in relation to gender, age, partner and immigrant status. J Rehabil Med. 2002;34:239-46.

38. Térémetz M, Colle F, Hamdoun S, Maier MA, Lindberg PG. A novel method for the quantification of key components of manual dexterity after stroke. J Neuroeng Rehabil. 2015;12:64.

39. Shumway-Cook A, Woollacott M. Motor control: translating research into clinical practice. Philadelphia: Lippincott Williams \& Wilkins; 2012.

40. Meyer S, Verheyden G, Brinkmann N, Dejaeger E, De Weerdt W, Feys H, et al. Functional and motor outcome 5 years after stroke is equivalent to outcome at 2 months: follow-up of the collaborative evaluation of rehabilitation in stroke across Europe. Stroke. 2015;46:1613-9.

41. Faria-Fortini I, Michaelsen SM, Cassiano JG, Teixeira-Salmela LF. Upper extremity function in stroke subjects: relationships between the international classification of functioning, disability, and health domains. J Hand Ther. 2011:24:257-64.

\section{Submit your next manuscript to BioMed Central and we will help you at every step:}

- We accept pre-submission inquiries

- Our selector tool helps you to find the most relevant journal

- We provide round the clock customer support

- Convenient online submission

- Thorough peer review

- Inclusion in PubMed and all major indexing services

- Maximum visibility for your research

Submit your manuscript at www.biomedcentral.com/submit 